\title{
Multiple strictures of the small intestine demanding surgical intervention as a rare consequence of graft versus host disease - case report
}

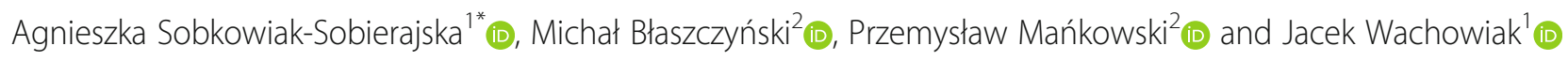

\begin{abstract}
Background: Intestinal strictures are an extremely rare and life-threating complication in patients undergoing allogenic hematopoietic stem cell transplantation (allo-HSCT). We describe the case of a child operated for multiple strictures of the small intestine secondary to GvHD.

Case presentation: A 7-year-old girl underwent second allo-HSCT from matched unrelated donor for post-CALRpositive-ET myelofibrosis and its relapse after the first HSCT. HSCT was complicated with grade IV acute GvHD (skin and gut). The patient was treated with CsA, with methylprednisolone, and due to corticosteroid resistance subsequently with etanercept and ECP. She required parenteral nutrition due to malabsorption symptoms and anorexia for 15 months after HSCT. On day +457 , the laparotomy was performed due to increasing symptoms of mechanical bowel obstruction. Ten critical cicatricial strictures of the small intestine were revealed. Nine segmental resections of the strictured segments and 6 intestinal anastomoses were done during one surgical procedure. The postoperative period was uncomplicated, and oral feeding was gradually implemented, achieving full coverage. Pathological examination confirmed the diagnosis of overlap syndrome of acute and chronic GvHD. Therefore, the patient started therapy with ruxolitinib which was continued till 30 months after HSCT. Corticosteroids were gradually withdrawn on day +574 . Currently, 32 months after the second HSCT, no GvHD and no gastrointestinal disturbances are observed. The patient remains in hematological and molecular remission of myelofibrosis.
\end{abstract}

Conclusions: GvHD therapy is largely based on conservative treatment, but a patient presenting with occlusive symptoms due to permanent changes in the gastrointestinal wall needs to undergo surgery to ensure its efficient functioning.

Keywords: Graft versus host disease, Allogenic hematopoietic stem cell transplantation, Multiple strictures, Small intestine, Surgery, Pediatrics, MRI

\section{Background}

Acute graft-versus-host disease (aGvHD) affects approximately $40 \%$ of all recipients of allo-HSCT (allogenic hematopoietic stem cell transplantation). It can involve

\footnotetext{
* Correspondence: agnieszka.sobkowiaksobierajska@ump.edu.pl 'Department of Pediatric Oncology, Hematology and Transplantology, Poznan University of Medical Sciences, Szpitalna 27/33, 60-572 Poznan, Poland

Full list of author information is available at the end of the article
}

the skin, liver, and gastrointestinal (GI) tract. Common manifestations of GI-aGvHD are anorexia, nausea, vomiting, and diarrhea-typically green and watery. In severe case, diarrhea contains fresh blood and pieces of mucosa and is accompanied by abdominal cramps and, on occasion, paralytic ileus [1]. Moderate to severe aGvHD requires systemic therapy-high-dose methylprednisolone is considered to be a first-line treatment. Failure to respond to standard steroid doses (defined as

\section{Springer Open}

(ㅇ The Author(s). 2020 Open Access This article is licensed under a Creative Commons Attribution 4.0 International License, which permits use, sharing, adaptation, distribution and reproduction in any medium or format, as long as you give appropriate credit to the original author(s) and the source, provide a link to the Creative Commons licence, and indicate if changes were made. The images or other third party material in this article are included in the article's Creative Commons licence, unless indicated otherwise in a credit line to the material. If material is not included in the article's Creative Commons licence and your intended use is not permitted by statutory regulation or exceeds the permitted use, you will need to obtain permission directly from the copyright holder. To view a copy of this licence, visit http://creativecommons.org/licenses/by/4.0/. 
progression within 3-5 days of starting treatment or an incomplete response by 7-14 days) or recurrence after initial dose reduction (steroid dependence) necessitate second-line treatment. The most effective therapy for steroid refractory aGvHD is not established. The history and severity of aGvHD remains by far strongest predictor of the development of chronic GvHD (cGvHD), which counts for approximately $25 \%$ of late non-relapse mortality after allo-HSCT [2]. GI manifestations of cGvHD can lead to dysphagia, nausea, and vomiting or chronic diarrhea and malabsorption syndrome with subsequent malnutrition. Small bowel obstruction as a symptom of GI cGvHD is extremely uncommon. There are only a few case reports of this complication in pediatric patients after allo-HSCT, and therefore, its optimal treatment remains controversial.

We report a rare case of multiple small bowel strictures in a pediatric patient with the overlap syndrome of steroid-resistant acute and chronic GvHD in the GI tract cured thanks to successful surgical intervention.

\section{Case presentation}

A 6-year-old girl was diagnosed with CALR-positive myelofibrosis secondary to essential thrombocythemia (ET). She underwent allogenic bone marrow (BM) transplantation from unrelated 10/10 matched donor. The conditioning regimen consisted of treosulfan $\left(12 \mathrm{~g} / \mathrm{m}^{2}\right.$ intravenously for 3 days), fludarabine $\left(30 \mathrm{mg} / \mathrm{m}^{2}\right.$ intravenously for 5 days), thiotepa $(2 \times 5 \mathrm{mg} / \mathrm{kg}$ intravenously for 1 day), and anti-thymocyte globulin (Thymoglobulin $2.5 \mathrm{mg} / \mathrm{kg} /$ day intravenously for 3 days). Cyclosporin A (CsA) and short-course methotrexate $\left(10 \mathrm{mg} / \mathrm{m}^{2}\right.$ intravenously on day $+1,+3,+6$ ) were administered for GvHD prophylaxis. CsA was started 1 day before HSCT. The dose of nucleated cells infused was $3.0 \times 10^{8} / \mathrm{kg}$ and the dose of CD34+ cells was $3.0 \times 10^{6} / \mathrm{kg}$. The platelet count achieved stable value above $20 \times 10^{9} / \mathrm{L}$ on day + 32 after transplantation, and on day +34 , the absolute neutrophil count (ANC) rose above $0.5 \times 10^{9} / \mathrm{L}$. No symptoms or signs of acute GVHD were observed. The post-transplant period was complicated with EBV reactivation. On day +98 , recurrent mutation in calreticulin $(C A L R)$ gene and mixed donor chimerism (66\% donor DNA) were detected. On day +135 , donor lymphocyte infusion at a dose of $1 \times 10^{7} \mathrm{CD} 3+$ cells $/ \mathrm{kg}$ was performed, but in the lack of the expected effect of DLI, the patient was finally qualified to the second allo-HSCT from the same donor. Second HSCT was performed 7 months after the first one. The conditioning regimen consisted of busulfan $(4.4 \mathrm{mg} / \mathrm{kg}$ intravenously for 4 days), fludarabine $\left(30 \mathrm{mg} / \mathrm{m}^{2}\right.$ intravenously for 5 days), melphalan $\left(140 \mathrm{mg} / \mathrm{m}^{2}\right.$ intravenously for 1 day), and anti-thymocyte globulin (ATGAM $12 \mathrm{mg} / \mathrm{kg} /$ day intravenously for 3 days). CsA (from day - 1) and methotrexate $\left(10 \mathrm{mg} / \mathrm{m}^{2}\right.$ intravenously only on day +1 due to severe mucositis) were administered for GVHD prophylaxis. The dose of transplanted CD34+ cells was $2.76 \times 10^{6} / \mathrm{kg}$. On day +12 , after transplantation, the ANC rose above $0.5 \times 10^{9} / \mathrm{L}$, and the platelet count achieved stable value above $20 \times 10^{9} / \mathrm{L}$ on day +14 . On day +13 , the complete donor chimerism in peripheral blood was detected.

On day +8 , maculopapular rash, diarrhea, and abdominal pain occurred. The patient was diagnosed with aGvHD grade II, and its treatment with methylprednisolone (MP) at a dose $1 \mathrm{mg} / \mathrm{kg} /$ day intravenously was started. As GvHD symptoms persisted, MP dose was increased to $2 \mathrm{mg} / \mathrm{kg} /$ day on day +10 . On day +24 , the rash and abdominal pain worsened-MP dose was again increased to $2 \mathrm{mg} / \mathrm{kg}$. The diagnosis of steroid-refractory GvHD grade IV was established, and the second-line treatment with anti-thymocyte globulin (Thymoglobulin) was administered at a total dose $10 \mathrm{mg} / \mathrm{kg}$. On day +34 , skin lesions decreased, but abdominal pain and bloody diarrhea persisted. The child required intensive analgesic treatment, including morphine, and continuous albumin supplementation due to severe enteropathy. On day + 42 , oral budesonide was added to the treatment. The patient required terlipressin for several days because of severe gastrointestinal bleeding. Adenovirus (ADV) PCR assay in the stool performed on day +43 was positive. The child received two doses of cidofovir; the dose of MP was reduced simultaneously. Despite the treatment, an increase in the amount of ADV copies in the stool and no improvement in gastrointestinal symptoms were observed. Endoscopy of the gastrointestinal tract showed intense inflammatory and erosive lesions in the duodenum and stomach, as well as inflammation in the colon mucous membrane. The patient periodically presented signs of paralytic gastrointestinal obstruction. On day + 120, extracorporeal photopheresis (ECP) procedures were started (off-line method using Optia Spectra and UVA-PIT system) twice weekly. After 4 weeks of treatment, a reduction in the severity of diarrhea and abdominal pain was observed, and the patient gradually began oral nutrition. ADV negativity in the stool was also obtained. During the attempt of steroid dose reduction, an increased abdominal pain and bloody stools occurred again. Since day +170 , the patient presented with several episodes of paralytic obstruction. Gastroscopy performed on day +196 revealed less severe inflammatory lesions in the duodenum and stomach, and CSA dose reduction was started until its complete withdrawal on day +225 . The patient continued ECP and steroid treatment. On day +266 , follow-up endoscopic examinations showed persistent inflammatory changes in the upper and lower gastrointestinal tract. On day +272 , an upper and lower gastrointestinal tract contrast study showed 
stomach atony and no strictures in the intestines. Cisapride treatment was started with the improvement in oral fluid and food intake. The patient continued steroid (MP $0.5 \mathrm{mg} / \mathrm{kg} /$ day) and ECP treatment and was discharged home on day +304 .

On day +382 , the patient presented with significantly increased C-reactive protein and procalcitonin level, significant dyselectrolytemia, leukocytosis, and thrombocytopenia. Abdominal ultrasound revealed small intestine loops segmentally widened to approx. $30 \mathrm{~mm}$, with a thickened wall up to $4 \mathrm{~mm}$. Broad spectrum antibiotic therapy and intravenous steroid treatment were started-the child's clinical condition improved.

On day + 412, due to GI GvHD exacerbation etanercept $(0,4 \mathrm{mg} / \mathrm{kg}$ twice weekly) was added to the therapy and the child received its 8 doses. ECP treatment was continued till day +435 (74 ECP procedures were done in total).

On day +440 , the child again presented with abdominal obstruction symptoms: acute abdominal pain and vomiting. In the physical examination, the abdomen was soft, moderately painful with loud and vivid peristalsis and visible bowel loops. Abdominal ultrasound showed intestinal loops strongly widened with a diameter of up to $5.2 \mathrm{~cm}$ with residual food content and ineffective pendulum peristalsis. Abdominal $\mathrm{X}$ ray showed signs of $\mathrm{ab}-$ dominal obstruction. The consulting surgeons recommended conservative treatment (total parenteral nutrition, antibiotic therapy, enemas, analgetics, increased methylprednisolone dose $-1 \mathrm{mg} / \mathrm{kg}$ ) but the patient's status was getting worse. Upper and lower gastrointestinal tract contrast study revealed small bowel obstruction. MRI (magnetic resonance imaging) showed: significantly widened small intestine loops up to $5.0 \mathrm{~cm}$, with fluid levels-features of obstruction; in the middle abdomen and lower abdomen, loops of the small intestine with a thickened wall up to $5 \mathrm{~mm}$, with narrow lumen and strong contrast enhancement. The child was qualified and prepared to the surgical treatment. On day + 456, laparotomy was performed.

Surgery was done using right paramedian laparotomy. Distended intestinal loops filled with liquid and gaseous contents were explored proximally to critical stenoses in small intestine. Totally ten stenotic sites of small intestine were found localized at $80 \mathrm{~cm}$ below ligament of Treitz and distending segmentally to ileum terminale at $25 \mathrm{~cm}$ above Bauchin's valve. Nine segmental resections of small intestine were performed with six intestinal anastomoses (in four using GIA stapling device), and one stenotic site was incised longitudinally with transverse anastomosis (Figs. 1 and 2). Reassuming $45 \mathrm{~cm}$ of all intestinal segments were removed.

After the surgery oral nutrition was gradually increased. Postoperative course was uneventful and patient

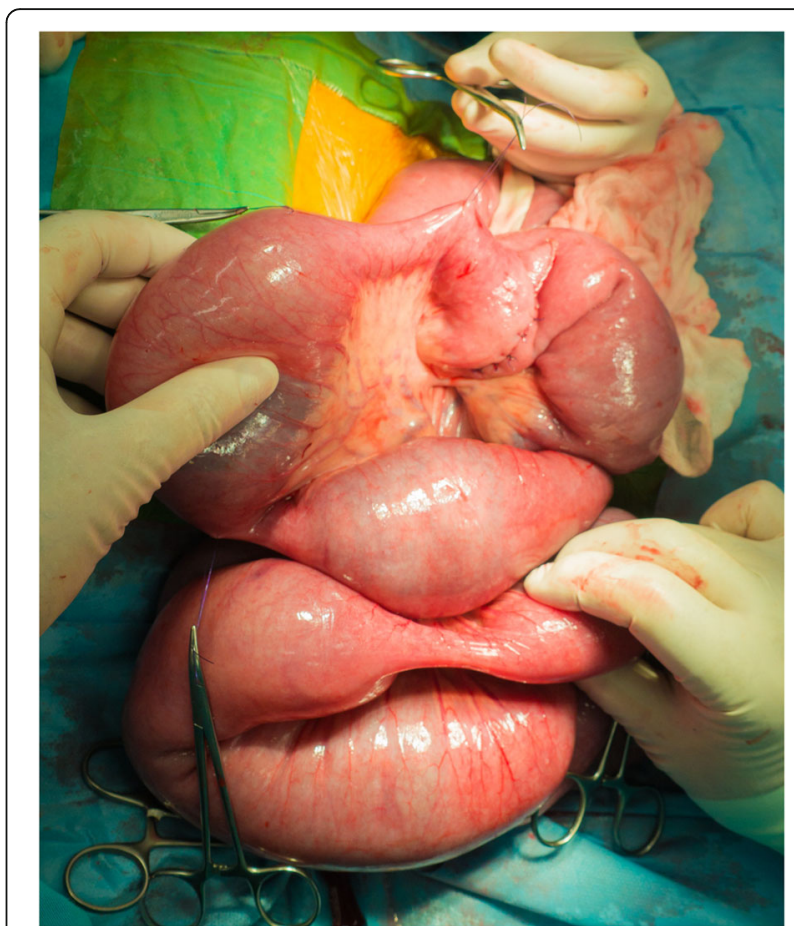

Fig. 1 Intraoperative view: two stenotic segments of small intestine and side-to-side anastomosis completing intestinal resection

recovered in 3 weeks. Due to child's improving clinical condition, the dose of methylprednisolone was reduced from 1.5 to $0.4 \mathrm{mg} / \mathrm{kg}$. Pathological examination of the resected parts of the intestine confirmed the diagnosis of overlap syndrome of acute and chronic GvHD. The inner diameters of stenotic sites ranged from 3 to 15 $\mathrm{mm}$. Therefore, the patient started therapy with ruxolitinib at a dose of $2.5 \mathrm{mg}$ per day on day +484 , while still receiving methylprednisolone at a dose of $0.35 \mathrm{mg} / \mathrm{kg}$ b.w. per day. Corticosteroid therapy was withdrawn on day +574. The patient continued treatment with

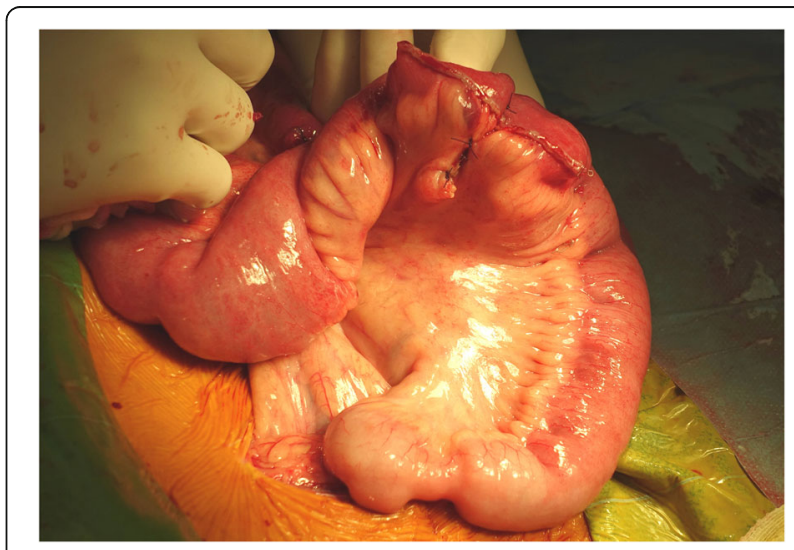

Fig. 2 Intraoperative view: side-to-side intestinal anastomosis with GIA stapler and distal intestinal stenosis 
ruxolitinib till 30 months after HSCT. Currently, 32 months after the second HSCT, no recurrence of GvHD and no gastrointestinal disturbances are observed. The patient remains in hematological and molecular remission of post-CALR-positive ET myelofibrosis.

\section{Discussion}

The GI tract is commonly affected after HSCT by conditioning regimen-related toxicity, infections of various etiology, and acute as well as chronic GvHD. Among these complications neutropenic colitis, infectious colitis and GvHD are the most common. Most of these complications are successfully treated, but steroid-resistant GvHD (SR-GvHD) is still a challenge for transplantologists and correlates with higher morbidity and mortality. There is no generally accepted second-line treatment for SRGvHD. Most of therapeutical options are immunosuppressive agents. An increase in immunosuppression correlates with a higher risk of infection that was observed in our patient (persistent ADV reactivation, pneumonia). Therefore, ECP, with its immunomodulatory rather than immunosuppressive effect, seems to be a good therapeutic option for such patients. In an Italian retrospective study of pediatric patients treated with ECP for aGvHD, a complete response was obtained in $72 \%$ of patients, a partial response was observed in $11 \%$, and there was no response in $17 \%$ of patients [3]. The rate of complete response $(\mathrm{CR})$ in the different organs varies between studies [4]. In another systematic review of prospective studies on the ECP efficacy in the treatment of SR or SD acute and chronic GvHD [5], the overall response rate were $69 \%$ and $64 \%$ for acute and chronic GVHD, respectively. The best results were seen in skin and GI tract involvement. The inclusion of ECP therapy turned out to be crucial in our patient.

$\mathrm{ADV}$ is a known factor that may contribute to the inflammatory process in GvHD and may be involved in bronchiolitis obliterans and intussusception. ADV was isolated from the patient's stool, and its presence correlated with the severity of GI symptoms. It is not known how far it may participate in inflammation leading to the obliteration of the gut lumen. Gavel et al. [6] reported a case of a child with GvHD and subsequent bowel obstruction who had a history of CMV and ADV reactivation in the posttransplant period. ADV infection occurs in $32 \%$ of pediatric and $6 \%$ of adult transplant recipients [7]. Cidofovir is the most used antiviral therapy [8] in ADV reactivation, but it was not effective in our patient. It was only the reduction in immunosuppression after the start of ECP treatment that lead to the negativity of ADV in the stool.

It is worth considering whether type of therapy used in the treatment of GvHD may affect the risk of intestinal wall fibrosis development. Two of the immunosuppressive drugs used in our patient, CsA and etanercept, are known causes of fibrosis in other organs. CsA treatment may lead to CsA-induced tubulointerstitial renal fibrosis which is probably caused by a dose-dependent increase in transforming growth factor- $\beta$ secretion from proximal tubular cells [9]. We found no reports of an increased risk of gastrointestinal tract wall fibrosis associated with CsA treatment. The impact of anti-tumor necrosis factor alpha (TNF- $\alpha$ ) therapy (ex. etanercept) on the development of fibrosis is not fully understood. It is known that TNF- $\alpha$ counterbalances the effects of TGF- $\beta$ so the inhibition of TNF- $\alpha$ may promote the profibrotic signaling cascade. Some histopathological studies performed in patients with Crohn's disease who were treated with anti-TNF- $\alpha$ agents before surgery revealed a distinct pattern of submucosal hyaline fibrosis, with increased fibrosis in the muscularis mucosae and muscularis propria [10]. In our patient, the etanercept treatment preceded an episode of mechanical bowel obstruction by approximately 4 weeks although clinical symptoms of impaired GI tract permeability were observed before the start of this therapy.

Small bowel obstruction is an extremely rare complication of GvHD. Severe inflammation process in the GI tract may lead to mural necrosis which results in extensive fibrosis and subsequent obliteration of the gut. The therapeutic options include conservative methods - gastric decompression and total parenteral nutrition. While this approach becomes ineffective, surgery is to be considered. That decision is extremely difficult regarding rather poor general condition of the patient. The diagnostic measures to define the occlusive changes include plain upright abdominal radiography. In our opinion, upper and lower gastrointestinal contrast study presented with low value. Otherwise, MRI precisely determined the pathologic changes in involved intestines and delivered valuable information before surgery. In our opinion, MRI should be a method of choice in diagnosing patients with GI GvHD who present with abdominal obstruction symptoms especially if the symptoms persist or the episodes recur. In the literature, there are only a few reports on this occlusive complication and most of them concern pediatric patients. Among 8 pediatric patients with small bowel obstruction included in a systematic review made by Gutierrez et al. [11], pathology of the resected gut revealed ulceration with fibrosis in all but one; 3 had signs of persistent GVHD. In another series of 4 cases by Tordjman et al. [12], small bowel occlusion occurred 5-16 months after graft, and computed tomographic scan revealed multiple small intestinal stenoses. All these patients underwent surgical resection of affected loops and stigmata of acute and chronic GVHD features, as well as extensive ulcerations, were observed in all ileal specimens. The histopathology of the resected parts of the small intestine in our patient 
also revealed the overlap syndrome of acute and chronic GvHD. In most described cases, the follow-up after surgery showed successful outcome, so did our patient.

After the surgery, we started ruxolitinib therapy based on its effectiveness in SR-GvHD [13]. We observed no side effects except for mild anemia. This therapy allowed us to discontinue other immunosuppressive agents (cyclosporin and steroids) and finally, 12 weeks ago, also ruxolitinib therapy. No recurrence of GvHD and no gastrointestinal disturbances were observed at 32 months after the second HSCT. The patient remains in good general condition and in hematological and molecular remission of post CALR-positive ET myelofibrosis.

\section{Conclusions}

In patients demonstrating resistance to complex systemic GvHD treatment, the small bowel strictures may develop with subsequent life-threatening obstruction of the gastrointestinal tract. These patients may require urgent surgical intervention. MRI seems to be a valuable measure in preparing such patients to surgical treatment.

The impact of ADV reactivation in allo-HSCT patients on late complications in the GI tract requires further investigations.

\section{Abbreviations \\ aGvHD: Acute graft versus host disease; ADV: Adenovirus; allo- HSCT: Allogenic hematopoietic stem cell transplantation; ANC: Absolute neutrophil count; BM: Bone marrow; b.w.: Body weight; CALR: Calreticulin; CGvHD: Chronic graft versus host disease; CsA: Cyclosporine A; ECP: Extracorporeal photopheresis; ET: Essential thrombocythemia; Gl: Gastrointestinal; GvHD: Graft versus host disease; MP: Methylprednisolone; MRI: Magnetic resonance imaging; SR-GvHD: Steroid resistant graft versus host disease}

\section{Acknowledgements}

We would like to thank all the doctors and medical staff involved in the treatment process of our patient for their commitment.

\section{Authors' contributions}

A.S.: conception, design of the work, acquisition, interpretation of data. M.B. conception, design of the work, acquisition, interpretation of data, revision. P.M.: revision. J.W.: conception, interpretation of data, revision. All authors have read and approved the manuscript.

\section{Funding}

The authors have declared no funding.

\section{Availability of data and materials}

Data sharing is not applicable to this article as no datasets were generated or analyzed during the current study.

\section{Ethics approval and consent to participate}

Not applicable.

\section{Consent for publication}

Written consent from parents was obtained.

\section{Author details}

'Department of Pediatric Oncology, Hematology and Transplantology, Poznan University of Medical Sciences, Szpitalna 27/33, 60-572 Poznan, Poland. ${ }^{2}$ Chair and Department of Pediatric Surgery, Traumatology and Urology, Poznan University of Medical Sciences, Szpitalna 27/33, 60-572 Poznan, Poland

Received: 7 May 2020 Accepted: 1 September 2020

Published online: 01 November 2020

\section{References}

1. The EBMT Handbook 2019. EBMT and the Author(s) 2019. https://www.ebmt org/sites/default/files/2019-01/2019_Book_TheEBMTHandbook.pdf. 325 p.

2. Grube $M$, Holler E, Weber D, et al. Risk factors and outcome of chronic graft-versus-host disease after allogeneic stem cell transplantation-results from a single-center observational study. Biol Blood Marrow Transpl. 2016; 22:1781-91.

3. Calroe E, Marson P, Pillon M. Treatment of acute graft-versus-host disease in childhood with extracorporeal photochemotherapy/photopheresis: the Padova experience. Biol Blood Marrow Transplant. 2015;21:1963-72.

4. Nygaard M, Wichert S, Berlin G, Toss F. Extracorporeal photopheresis for graft-vs-host disease: a literature review and treatment guidelines proposed by the Nordic ECP quality group. Eur J Hematol. 2020;104:361-75.

5. Abu-Dalle I, Reljic T, Nishihori T, et al. Extracorporeal photopheresis in steroid-refractory acute or chronic graft-versus-host disease: results of a systematic review of prospective studies. Biol Blood Marrow Transplant. 2014;20:1677-86.

6. Gavel G, Marven S, Evans MJ, et al. Obliterative enteritis complicating graft versus host disease. Bone Marrow Transplant. 2003;32:1097-8.

7. Sedlacek P, Petterson T, Robin M. Incidence of adenovirus infection in hematopoietic stem cell transplantation recipients: findings from the AdVance study. Biol Blood and Marrow Transplant. 2019;25:810-8.

8. Matther-Martin S, Feuchtinger T, Shaw PJ. European guidelines for diagnosis and treatment of adenovirus infection in leukemia and stem cell transplantation: summary of ECIL-4 (2011). Transpl Infect Dis. 2012;14:55563.

9. Slattery C, Campbell E, McMorrow T, Ryan MP. Cyclosporine A-induced renal fibrosis: a role for epithelial-mesenchymal transition. Am J Pathol. 2005; 167(2):395-407. https://doi.org/10.1016/S0002-9440(10)62984-7.

10. Schaeffer DF, Walsh JC, Kirsch R, Waterman M, Silverberg MS, Riddell RH. Distinctive histopathologic phenotype in resection specimens from patients with Crohn's disease receiving anti-TNF-a therapy. Hum Pathol. 2014;45(9): 1928-35. https://doi.org/10.1016/j.humpath.2014.05.016.

11. Gutierrez CA, Raval MV, Vester HR. Surgical treatment of intestinal complications of graft versus host disease in the pediatric population: case series and review of literature. J Pediatr Surg. 2017;52:1718-22.

12. Tordjman M, Ouachee M, Bonnard A. Small bowel stenosis: a manifestation of chronic graft-versus-host disease in children? Hum Pathol. 2018;72:174-9.

13. Khoury HJ, Langston AA, Kota VK. Ruxolitinib: a steroid sparing agent in chronic graft-versus-host disease. Bone Marrow Transplant. 2018;53:826-31.

\section{Publisher's Note}

Springer Nature remains neutral with regard to jurisdictional claims in published maps and institutional affiliations. 